\title{
Fluctuating electric field particle acceleration at a magnetic field null point
}

\author{
Panagiota Petkaki* and Alexander L. MacKinnon ${ }^{\dagger}$ \\ * Physical Sciences Division, British Antarctic Survey, Cambridge, CB3 OET, UK \\ ${ }^{\dagger}$ DACE/Physics and Astronomy, University of Glasgow, Glasgow, G12 8QQ, UK
}

\begin{abstract}
Release of stored magnetic energy via particle acceleration is a characteristic feature of astrophysical plasmas. Magnetic reconnection is one of the primary candidate mechanisms for releasing non-potential energy from magnetized plasmas. A collisionless magnetic reconnection scenario could provide both the energy release mechanism and the particle accelerator in flares. We studied particle acceleration consequences from fluctuating electric fields superposed on an X-type magnetic field in collisionless hot solar plasma. This system is chosen to mimic generic features of dynamic reconnection, or the reconnective dissipation of a linear disturbance. Time evolution of thermal particle distributions are obtained by numerically integrating particle orbits. A range of frequencies of the electric field is used, representing a turbulent range of waves. Depending on the frequency and amplitude of the electric field, electrons and ions are accelerated to different degrees and often have energy distributions of different forms. Protons are accelerated to gammaray producing energies and electrons to and above hard X-ray producing energies in timescales of 1 second. The acceleration mechanism could be applicable to all collisionless plasmas.
\end{abstract}

Keywords: Flares; Magnetic Reconnection; Particle Orbits

PACS: $52.65 . \mathrm{Cc} ; 94.30 \mathrm{cp} ; 52.35 . \mathrm{Vd} ; 96.60 . \mathrm{Iv} ; 96.60 . \mathrm{qe}$

\section{INTRODUCTION}

Release of stored magnetic energy via particle acceleration is a characteristic feature of astrophysical plasmas. In the particular case of the Sun, we see this manifested in the catastrophic events of flares, as well as in quieter phenomena like radio noise storms. Solar flares present particular challenges to theory. A large fraction (several tens of percent) of the flare energy is manifested initially in the form of fast electrons (accelerated out of the background distribution to $\sim 100 \mathrm{keV}$ in about 1 second and to $\sim$ $100 \mathrm{MeV}$ in a few seconds), which reveal their presence by producing bremsstrahlung $\mathrm{X}$-rays [1]. Protons are accelerated in flares to energies of several tens of MeVs in a timescale of one second [2]. Thus the acceleration of particles is an important part of the energy release process, rather than an energetically unimportant consequence of the flare. Moreover, radio signatures (Type I noise storms, Type III bursts away from flares) testify to particle acceleration at "quiet" times.

Magnetic reconnection is one of the primary candidate mechanisms for releasing nonpotential energy from magnetized plasmas [3]. The electric field in the current-carrying region also makes it a natural particle accelerator. Martens ([4]) gave order-of-magnitude arguments in favor of a collisionless current sheet as both the energy release mechanism and the particle accelerator in flares. Particle acceleration is energetically the primary result of such a situation. Collisionless reconnection thus assumes great potential im- 
portance in understanding the flare process, particle acceleration, energy conversion and release in astrophysical plasmas generally (see [5] and references therein).

We present test particle calculations designed to illuminate the consequences for particle acceleration of dynamic reconnection. We have in mind the picture of Craig and McClymont ([6], [7]), in which a linear disturbance passes through a magnetic configuration containing an X-type neutral point. The disturbance travels without dissipating with the local Alfvén speed until it approaches the diffusion region surrounding the neutral point, where the resistive diffusion term in the induction equation becomes important. The wave damps resistively in a few system transit times, heating or accelerating particles.

In Petkaki and MacKinnon ([8]), we examined the behavior of protons in the presence of electric and magnetic fields obtained from the Craig and McClymont [6] analysis. Petkaki and MacKinnon [5], have explored the consequences of time-dependent reconnection in a parametric way that does not depend on a particular set of simplifying physical assumptions or boundary conditions. Here we revisit these calculations highlighting certain key points. We follow the particle evolution in the presence of simple fields chosen to mimic generic features of dynamic reconnection. Time-dependence of the electric field is the essential ingredient reflecting the dynamic character of the reconnection. Particularly relevant to our work is the exploratory, analytical study of Litvinenko [9] which looks at charged particle orbits in an oscillating electric field in a magnetic field containing a neutral line

We use the Craig and McClymont [6] linear solution as a qualitative guide for the spatial and temporal form of the electric field. Our adopted field also resembles a linear situation in displaying a time dependence that does not change (i.e. does not develop multiple frequencies, saturate, etc.). Our linear picture will provide useful insight of what happens in a flare and may be particularly relevant to non-flaring particle acceleration, e.g. in solar noise storms. Many previous studies of test particle evolution in steady reconnection exist. Recent work studies regular and chaotic dynamics in 3-D reconnecting current sheets (e.g. [10]) or studies particle orbits in the presence of 3-D magnetic nulls (e.g. $[11,12])$.

\section{PARTICLE ACCELERATION MODEL AND RESULTS}

We investigate the particle acceleration from fluctuating electric fields superposed on a $\mathrm{X}$-type magnetic field to mimic generic features of dynamic, collisionless reconnection [6]. We solve numerically the relativistic equations of motion of test particles in electromagnetic fields and in the observer's reference frame [5]. To model the reconnection magnetic field, we adopt an idealized 2-D magnetic field containing an X-type neutral point: $\underline{\mathrm{B}}=\frac{B_{0}}{D}(y \hat{x}+x \hat{y})$. The $\mathrm{X}$-line (neutral line) lies along the $\mathrm{z}$-axis. The field strength depends on position. We assume that the field has a value of $10^{2}$ gauss at a typical active region distance of $10^{9} \mathrm{~cm}$ from the neutral point, so $B_{0} / D=10^{-7}$ gauss $\mathrm{cm}^{-1}$. An electric field is imposed in the $z$ direction, with spatial and temporal form chosen to mimic qualitative features of dynamic reconnection and is given by the form $\underline{\mathrm{E}}=E_{0} \sin (\omega t) \hat{z} f(x, y)$ where $f(x, y)$ describes the spatial variation of $\underline{\mathrm{E}}$. We take $f(x, y)^{-}=\exp \left(-\alpha_{i} \sqrt{ }(|r|)\right)$ where, $\alpha_{p}=2.5 \times 10^{-1}$ for protons, $\alpha_{e}=3.776 \times 10^{-2}$ for 

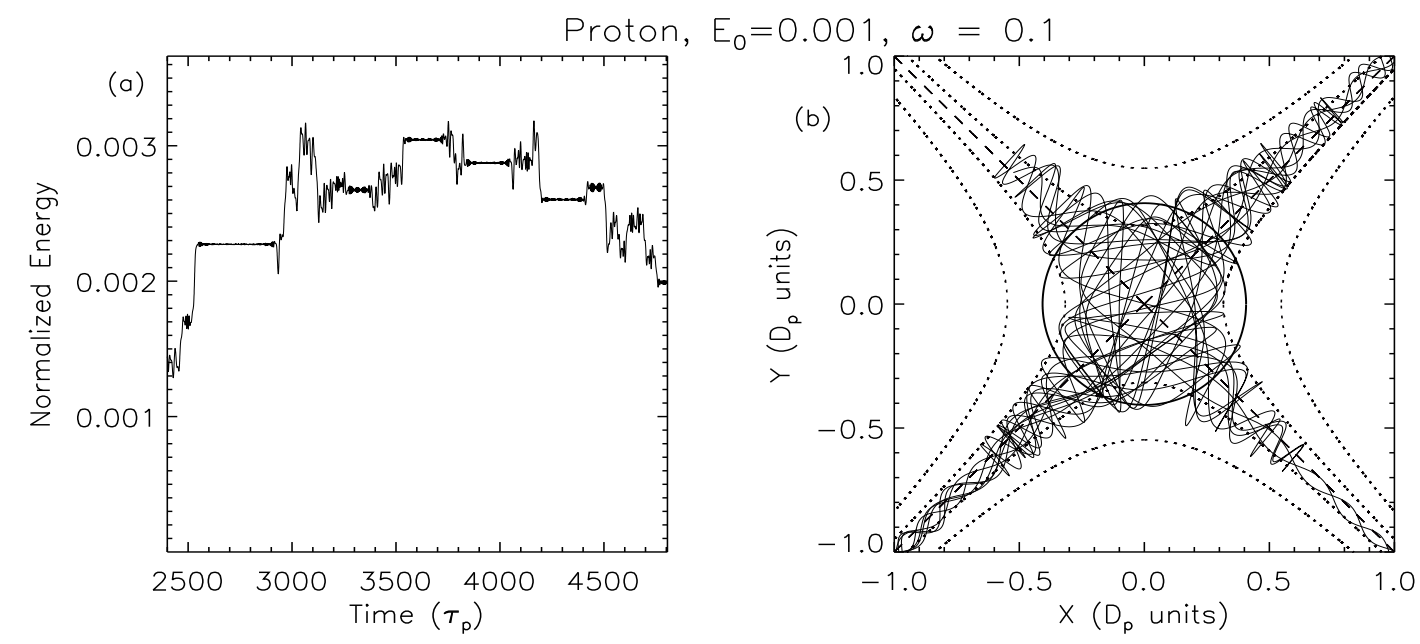

FIGURE 1. Proton orbit in time-varying electric field of $\omega=0.1$, with amplitude $\bar{E}_{0}=0.001$. (a) Energy (dimensionless) as a function of time. (b) Projection of the orbit in the X-Y plane.

electrons, $|r|=\sqrt{ }\left(x^{2}+y^{2}\right)$ (see Figure 1 of Petkaki and MacKinnon, [5]). The spatial variation $f(x, y)$ is a stretched exponential in $|r|$ (Sornette, [16]). We normalize velocities to the speed of light and this has the consequence that distances are normalized to different values $D_{e}=c \sqrt{ }\left(m_{e} D / e B_{0}\right)$ and $D_{p}=c \sqrt{ }\left(m_{p} D / e B_{0}\right)$ for electrons and protons respectively [5], such that $D_{e}=1.3 \times 10^{5} \mathrm{~cm}$ and $D_{p}=5.6 \times 10^{6} \mathrm{~cm}$. Energies are normalized to the particle rest mass energy so that kinetic energy in dimensionless units is just $K_{\text {kin }}=\gamma-1$. We integrate the particle orbits up to 230400 timesteps $\left(\tau_{e}\right)$ for electrons and $5360\left(\tau_{p}\right)$ for protons. With $B_{0} / D=10^{-7}$ and our form of dimensionless units these times correspond to 1 second for electrons and protons. The initial velocities of the particles are picked randomly from a Maxwellian distribution of temperature $5 \times 10^{6} \mathrm{~K}(\sim 431 \mathrm{eV})$, a typical coronal value. We consider only small values for $\bar{E}_{0}$, consistent with the passage of a disturbance in the linear regime [6]. Values of 0.0001, and 0.001 are used in the actual calculation. The value 0.001 corresponds to electric field $=5.88 \times 10^{-4}$ statvolt $/ \mathrm{cm}$.

The frequency of oscillation of the electric field $\omega$ is a free parameter. Each simulation uses one value of $\omega$. We take values of $\omega$ such that $1 / 1000<\omega<10000$, corresponding to real frequencies in the range $5 \mathrm{~Hz}$ to $5 \mathrm{MHz}$ (cf. the frequency range of waves from the base of the solar corona, probably in the range $0.01 \mathrm{~Hz}$ to $10 \mathrm{KHz},[17])$. Since we aim to emulate a linear situation we may pick our test particles from an isotropic, homogeneous distribution representing the background. This is in contrast to particle studies of nonlinear reconnection, where consistency demands consideration of the motion of particles into the dissipation region. Since we use a test particle approach, particles do not interact with each other, nor do they influence the background field. The particle distribution including the accelerated component may well be unstable to growth of various sorts of waves, but here we neglect this possibility. We also neglect radiation losses. In the solar corona this is not a serious neglect (even for $10 \mathrm{MeV}$ electrons the radiative energy loss time is $\sim 3000 \mathrm{~s}$ ), but elsewhere in the cosmos it could become 

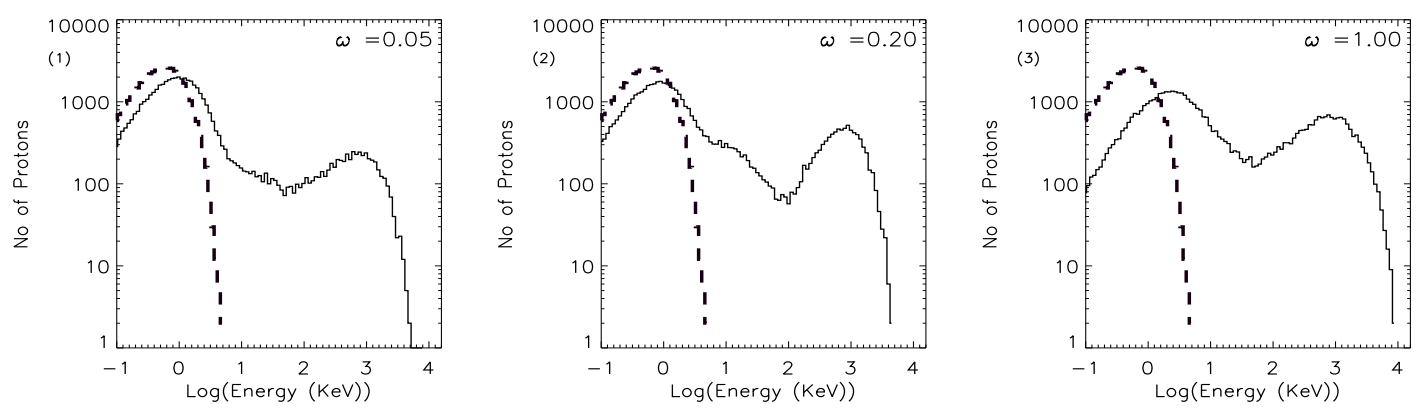

FIGURE 2. Proton distributions (full black line) for three frequencies of the electric field. The magnitude of the dimensionless electric field is $\bar{E}_{0}=0.001$. The total integration time is $5360 \tau_{p}$. Each distribution has 50000 test protons or electrons. We show the initial Maxwellian distribution in dashed lines in each panel.

significant.

The functional form of the nonadiabatic region as represented by the electric field form allows particles to gain or lose some energy randomly before returning to adiabatic motion and allows repeated encounters with the dissipation region. The magnetic mirroring in the extended magnetic configuration, results in a Fermi-type, 'stochastic' acceleration. A typical proton orbit which is shown in Figure 1. Close to the neutral point the gyroradius (Larmor radius) is not well defined since the particle is not bound to one magnetic field line and meandering motion is observed. The electric field accelerates or decelerates the proton causing further changes in the particle gyroradius and energy, thus resembling stochastic-type acceleration. Stochasticity is introduced by the phase of the electric field and the phase of the particle orbit and is sustained because of the form of the magnetic field [13]. Outside the magnetic neutral point area the particle mirrors and recrosses the non-adiabatic region and the process is repeated until the end of the integration time or until the particle escapes the outer boundary of the system. We see jets of accelerated particles along the separatrices.

The test particle calculation is numerically simpler than self-consistent approaches (e.g. Vlasov simulations, $[14,15]$ ) and gives useful insights to the particle energization process. In our model particle acceleration takes place for geometrical reasons. There is no threshold for this type of acceleration, unlike resonant interaction with low-frequency, MHD waves. Our results indicate that low-frequency waves may themselves perform the 'first-step' acceleration, if they propagate in a coronal structure including a neutral point. Sufficient number of pre-accelerated particles may be achieved if multiple neutral points are present.

Most of the resulting proton distributions have a bi-modal form like those in Fig. 2 (see also [5]). Electron distributions are also bi-modal for the highest frequencies, $20 \leq \omega \leq 500$ (see Figure 6 in [5]). Whereas for the lowest frequencies of the electric field the bulk of the initial electron Maxwellian distribution is accelerated, for the highest frequencies only part of the electron distribution is accelerated. Acceleration occurs for all frequencies $\omega \leq 10$ when addressing the proton distributions. The bi-modal form of the proton energy distributions might offer a way to have protons of gamma-ray 

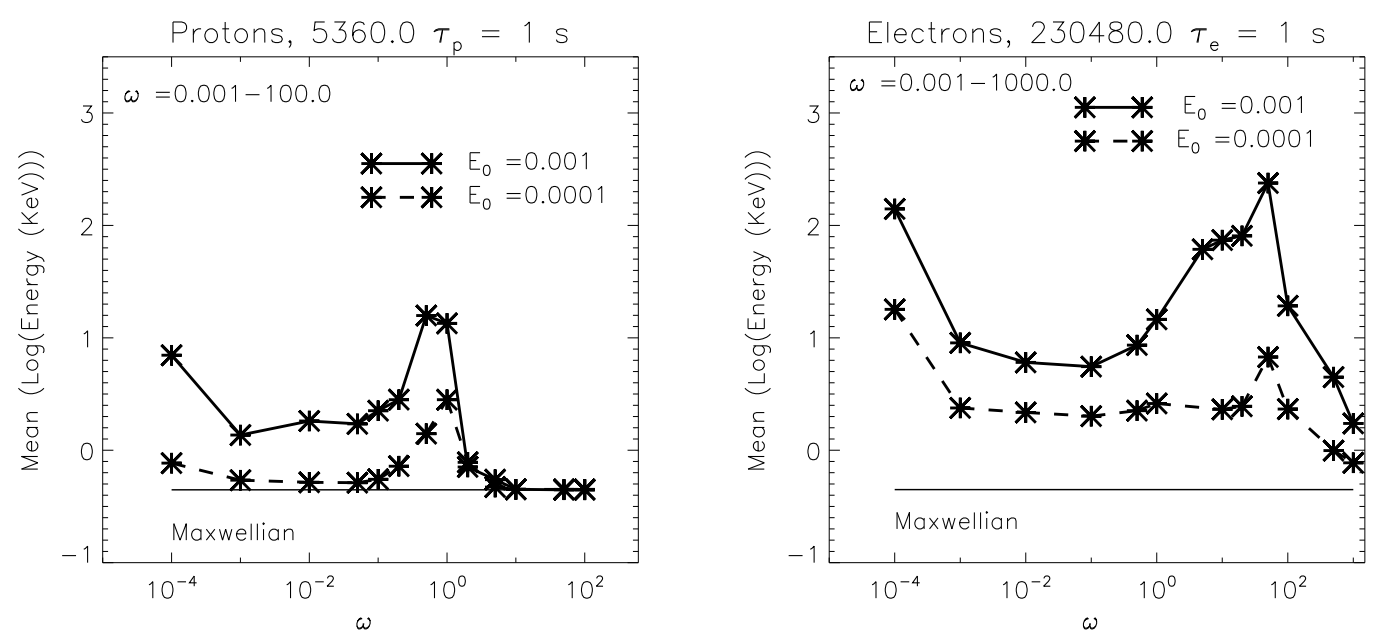

FIGURE 3. Mean Energy of proton and electron distributions for amplitude $\bar{E}_{0}=0.001$ and $\bar{E}_{0}=$ 0.0001 . The mean energy of the initial Maxwellian distribution is shown as a straight full line. The constant electric field case is represented by $\omega=0.0001$. The total integration time is 1 second.

producing energies $\left(K_{k i n} \geq 2 \mathrm{MeV}\right)$ without the energetically dominant population at lower energies as in a diffusive particle accelerator [18].

In Fig. 3 we plot the mean of the logarithm of the initial and final proton and electrons energy distributions versus the frequency of the electric field and for two amplitudes of the electric field $\bar{E}_{0}=0.0001$ (dashed star line), $\bar{E}_{0}=0.001$ (solid star line). We use the mean value of the logarithm of the energy to better represent the changes in highly non-thermal distributions. The effectiveness of acceleration of the two species varies according to the frequency of oscillation invoked. Electrons are accelerated for a broader spectrum of frequencies. The constant electric field case is represented by $\omega=0.0001$. Frequencies lower than 0.001 will also accelerate electrons as indicated by the net acceleration achieved for the constant electric field cases (Fig. 3), but frequencies higher than 1000 do not produce a net acceleration in the timescale of our model.

A local peak in the mean energy of the accelerated proton distribution is seen at $0.1<\omega<2.0$ (Fig. 3). The highest energy gain for the time-varying electric field is achieved when $0.2<\omega<2.0$, indicating a resonant acceleration process. This range of frequencies are comparable to the gyrofrequencies of protons in the adiabatic region for our set of initial conditions and to the proton inverse crossing time. Protons are accelerated for low electric field frequencies, achieving $\gamma$-ray producing energies in 5360 $\tau_{p}=1 \mathrm{~s}$ for frequencies $\omega<10$ and for $E_{0}=0.001$. Depending on the frequency of the electric field, $\sim 0.2 \%$ to $\sim 17.9 \%$ of the proton distributions get accelerated to $\gamma$-ray producing energies in $1 \mathrm{~s}$. For frequency $\omega=10$ and greater the energy distribution does not change significantly.

Considered as a function of $\omega$, the mean energy of the accelerated electron distribution exhibits a peak in the broad range $5<\omega<100$ (Fig. 3). Such a peak indicates a resonance involving two or more of the timescales in the problem. The initial gyrofrequencies of electrons lying in the adiabatic portion of the dissipation region also generally lie in this range. Inverse crossing times $\left(1 / t_{c r}\right)$, (see Equation 14 of [5]) com- 
parable with $\omega$ might also lead to enhanced acceleration. Using inverse crossing times, but taking account also of the mean increase in $u_{x, y}$ we do indeed find upper limits in the range $5<1 / t_{c r}<100$. For $E_{0}=0.001$ and for most frequencies of the electric field (and for constant electric field) the bulk of the electron distributions get accelerated to X-ray producing energies in $1 \mathrm{~s}$. When $E_{0}=0.0001$ and $\omega=50$, approximately $23 \%$ of the electron distribution accelerates to $\mathrm{X}$-ray producing energies.

We have shown that protons and electrons gain relativistic energies in times $\leq 1 \mathrm{~s}$, for fluctuating electric fields, for small electric field amplitudes and active region magnetic fields (see also [8]). Real time-dependent reconnection fields will have more general time-dependence but will possibly be expected to show some of the behavior found here. The variability of the effectiveness of acceleration of the two species according to the frequency of electric field oscillation might bear on the apparent variation of electron/proton ratios in flares and the phenomenon of 'electron-rich' flares. We note that higher frequency disturbances favor electrons over ions. Our calculations may give insight into particle acceleration in flares, and are also possibly relevant to quiescent, long-lasting phenomena such as radio noise storms $[19,5]$. Electrons accelerated at a neutral point will likely encounter very large mirror ratios, trapping them in the corona and accounting for the exclusively coronal phenomena accompanying noise storms.

\section{REFERENCES}

1. MacKinnon, A. L., 2006, in N. Gopalswamy, R. Mewaldt and J. Torsti (eds.), Solar Eruptions and Energetic Particles, AGU

2. Aschwanden M.J., 2002, Space Sci. Rev., 101, 1

3. Priest E.R. and Forbes T.A., 2001, Magnetic Reconnection: MHD Theory and Applications, Cambridge University Press, Cambridge

4. Martens P.C.H., 1988, ApJ, 330, L131

5. Petkaki P., MacKinnon A. L., 2007, A\&A, 472, 623, doi:10.1051/0004-6361:20066961

6. Craig I.J.D., McClymont A.N., 1991, ApJ, 371, L41

7. Craig I.J.D., McClymont A.N., 1993, ApJ, 405, 207

8. Petkaki P., MacKinnon A. L., 1997, Sol. Phys., 172, 279-286

9. Litvinenko Y.E. 2003, Solar Physics, 216, 189

10. Gontikakis C., Efthymiopoulos C., Anastasiadis A., 2006, MNRAS, 368, 293-304

11. Heerikhuisen, J., Litvinenko, Y.E. and Craig, I.J.D., 2002, Apj, 566,512-520,doi:10.1086/337957

12. Zharkova, V. V., Gordovskyy, M., 2004, ApJ, 604, 884-891

13. Martin R.F., 1986, JGR, 91, 11985

14. Petkaki, P., C. E. J. Watt, R. B. Horne, and M. P. Freeman, 2003, JGR, 108, 1442, doi: 10.1029/2003JA010092

15. Petkaki, P., M. P. Freeman, T. Kirk, C. E. J. Watt, and R. B. Horne, 2006, JGR, 111, A01205, doi:10.1029/2004JA010793

16. Sornette D., 2000, Critical phenomena in Natural sciences, Springer-Verlag, Berlin Heidelberg

17. Marsch E.K., Goertz C.K., Richter K., JGR, 87,5030-5044, 1982

18. MacKinnon A.L., 1991, Vistas Astron., 34, 331

19. Crosby, N., Vilmer, N., Lund, N., Klein, K.-L., Sunyaev, R., 1996, Sol. Phys. 167, 333 